\title{
Decreased serum albumin level indicates poor prognosis of COVID-19 patients: hepatic injury analysis from 2,623 hospitalized cases
}

\author{
Wei Huang ${ }^{1,2 \dagger}$, Chenze $\mathrm{Li}^{1 \dagger}$, Zhiquan Wang ${ }^{2}$, Hong Wang ${ }^{1}$, Ning Zhou ${ }^{1}$, Jiangang Jiang ${ }^{1}$, Li Ni ${ }^{1}$, \\ Xin A. Zhang ${ }^{3}$ \& Dao-Wen Wang ${ }^{1 *}$ \\ ${ }^{1}$ Division of Cardiology, Department of Internal Medicine, and Hubei Key Laboratory of Genetics and Molecular Mechanisms of Cardiological \\ Disorders, Tongji Hospital, Tongji Medical College, Huazhong University of Science and Technology, Wuhan 430010, China; \\ ${ }^{2}$ Department of Cardiology, Chinese PLA Central War Command General Hospital, Wuhan 430010, China; \\ ${ }^{3}$ Stephenson Cancer Center and Department of Physiology, University of Oklahoma Health Sciences Center, Oklahoma City, OK 73104, USA
}

Received April 20, 2020; accepted May 16, 2020; published online June 18, 2020

\begin{abstract}
Coronavirus disease 2019 (COVID-19) is a global pandemic which has caused numerous deaths worldwide. The present study investigated the roles of hypoproteinemia in the clinical outcome and liver dysfunction of COVID-19 patients. In this retrospective study, we extracted data from 2,623 clinically confirmed adult COVID-19 patients ( $\geq 18$ years old) between January 29 , 2020 and March 6, 2020 in Tongji Hospital, Wuhan, China. The patients were divided into three groups-non-critically ill, critically ill, and death groups - in accordance with the Chinese Clinical Guideline for COVID-19. Serum albumin, low-density lipoproteins cholesterol (LDL-C), and high-density lipoproteins cholesterol (HDL-C) concentrations and inflammatory cytokines levels were measured and compared among these three groups. The median age of these 2,623 patients was 64 years old (interquartile range (IQR), 52-71). Among the patients enrolled in the study, 2,008 (76.6\%) were diagnosed as non-critically ill and $615(23.4 \%)$ were critically ill patients, including $383(14.6 \%)$ critically ill survivors and $232(8.8 \%)$ critically ill deaths in the hospital. Marked hypoalbuminemia occurred in $38.2 \%, 71.2 \%$, and $82.4 \%$ patients in non-critically ill, critically ill, and death groups, respectively, on admission and $45.9 \%, 77.7 \%$, and $95.6 \%$ of these three groups, respectively, during hospitalization. We also discovered that serum low-density lipoprotein (LDL) and HDL levels were significantly lower in critically ill and death groups compared to non-critically ill group. Meanwhile, the patients displayed dramatically elevated levels of serum inflammatory factors, while a markedly prolonged activated partial thromboplastin time (APTT) in critically ill patients reflected coagulopathy. This study suggests that COVID-19-induced cytokine storm causes hepatotoxicity and subsequently critical hypoalbuminemia, which are associated with exacerbation of disease-associated inflammatory responses and progression of the disease and ultimately leads to death for some critically ill patients.
\end{abstract}

\section{albumin, hepatic injury, COVID-19}

Citation: Huang, W., Li, C., Wang, Z., Wang, H., Zhou, N., Jiang, J., Ni, L., Zhang, X.A., and Wang, D.W. (2020). Decreased serum albumin level indicates poor prognosis of COVID-19 patients: hepatic injury analysis from 2,623 hospitalized cases. Sci China Life Sci 63, 1678-1687. https://doi.org/ $10.1007 / \mathrm{s} 11427-020-1733-4$

†Contributed equally to this work

*Corresponding author (email: dwwang@tjh.tjmu.edu.cn) 


\section{INTRODUCTION}

Severe acute respiratory syndrome coronavirus 2 (SARSCoV-2) emerged in December, 2019 (Wilder-Smith et al., 2020), and today, it has become a global pandemic which killed thousands of patients. SARS-CoV-2 shares a close phylogenetic similarity to SARS-CoV (Liu et al., 2020a). Recent studies suggest that SARS-CoV-2 infection, e.g., coronavirus disease 2019 (COVID-19), mimics clinical phenotypes of SARS in its severity, including severe pneumonia and high mortality (Arentz et al., 2020; Holshue et al., 2020; Matthay et al., 2020; Peiris et al., 2004). However, it exhibits much greater contagiousness and special clinical virulence, such as higher chances of transmission between humans (Chen et al., 2020; Guan et al., 2020; Shi et al., 2020), multiple organ injury especially cardiac injuries in considerable number of patients (Chen et al., 2020; Chen et al., 2020), immune system dysfunction (Liu et al., 2020b; Saghazadeh and Rezaei, 2020), and many more. However, it is not clear whether the liver is injured in COVID-19 patients; moreover, its clinical phenotype and its association with clinical outcome are uncertain. In the present study, we have extracted the clinical data of 2,623 patients with COVID-19, identified the cause for reduction in serum proteins, and established the association of such reductions with clinical performance and prognosis of these patients.

\section{RESULTS}

\section{Presenting characteristics}

Figure 1 illustrates a flowchart of patient recruitment. Briefly, the study population included 2,705 hospitalized patients with clinically confirmed COVID-19, which, at the time of writing this manuscript, was the largest COVID-19 cohort. Among these patients, 18 had a history of renal dysfunction, eight had a history of cardiac dysfunction, and two had an acute myocardial infarction within one month. Twenty-three patients were younger than 18 years old, and 31 patients lack clinical information. Finally, 2,623 patients were recruited and 82 patients were excluded. In this study population, the median age was 64 (interquartile range (IQR), 52-71) years old, and 50\% were women. Compared to non-critically ill patients, critically ill patients were more likely to be older male individuals with a higher prevalence of hypertension, diabetes mellitus, coronary heart disease, malignancy, and chronic obstructive pulmonary disease. The proportion of patients complicated with at least one coexisting disease was significantly higher in the critically ill (65\%) and death groups $(69 \%)$ compared to the non-critically ill group $(42.9 \%)$. The baselines of heart rate and respiratory rate were higher in the critically ill and death groups, while the mean arterial pressure and body mass in- dex (BMI) had no significant differences among the three groups. We summarized the baseline clinical characteristics of patients according to clinical outcomes in Table 1.

\section{COVID-19 patients' albumin and lipid synthesis decreased as the severity of the disease progressed}

The proportion of the patients with decreased albumin levels on the first laboratory test was $46.9 \%$, and this proportion increased to $54.7 \%$ on the second test after hospitalization (Figure 2A). The proportions of patients with decreased albumin levels were $38.2 \%, 71.2 \%$, and $82.5 \%$ in the noncritically ill, critically ill and the death group, respectively, on the first laboratory test, and this proportion increased to $45.9 \%, 77 \%$, and $95.6 \%$ after hospitalization (Figure 2B). The average levels of serum albumin decreased in all three groups. During admission, the albumin was 36.60 (IQR, $33.18,40.40$ ) in the non-critically ill group, 32.20 (IQR, $29.60,35.75$ ) in the critically ill group, and 31.10 (IQR, $27.85,34.20)$ in the death group. In a multivariable adjusted model (age, sex, and comorbidities), $1 \mathrm{~g} \mathrm{~L}^{-1}$ decrease in albumin was associated with higher risk of progressing to critical stage and death (critically ill vs. non-critically ill, adjusted odds ratio (OR), 1.18; 95\% confidence interval (CI), $1.15-1.20 ; P=0.005$; death vs. non-critically ill, adjusted $\mathrm{OR}$, $1.25 ; 95 \% \mathrm{CI}, 1.20-1.30 ; P=0.008)$. In the second test, albumin significantly decreased to a median of 35.50 (IQR, $32.4,38.5)$ in the non-critically ill group, median of 31.30 (IQR, 28.80, 34.52) in the critically ill group, and median of 28.80 (IQR, 28.80, 31.35) in the death group (Figure 2C). After adjustments, the OR was $1.23(1.19-1.28 ; P=0.018)$ for critically ill vs. non-critically ill group and $1.47(1.37-1.54$; $P=0.029)$ for death vs. non-critically ill group. Such progressive decline of serum albumin was suggestive of poor prognosis. The level of albumin in the death group was significantly lower (median, 31.05 (IQR, 27.98, 34.23)) than that in the survival patients (median, 35.90 (IQR, 32.20, $39.80)$ ). As the death group was originally derived from the critically ill group, the level of albumin in this combined group (critically ill+death) was 31.90 (IQR, 27.85, 35.75), which was obviously lower than the non-critically ill group (median, 36.60 (IQR, 33.18, 40.40)). The ratio of albumin (ALB)/globulin (GLO) was also significantly lower in the death group (median, $0.84(0.74,0.98))$ and critical group (median, $0.93(0.79,1.10))$ compared to that in the noncritically ill group (median, $1.16(0.96,1.38)$ ) (Figure 2D). Meanwhile, the total protein also showed significant reduction in the critically ill and death group compared to the noncritically ill group (Figure 2E). However, the GLO increased with the severity of the disease, which especially became significantly higher in the death group 35.70 (IQR, 31.67, 39.05 ) in contrast to those in the survival groups (median, 32.10 (IQR, 28.80, 35.90)) (Figure 2F). The significant re- 


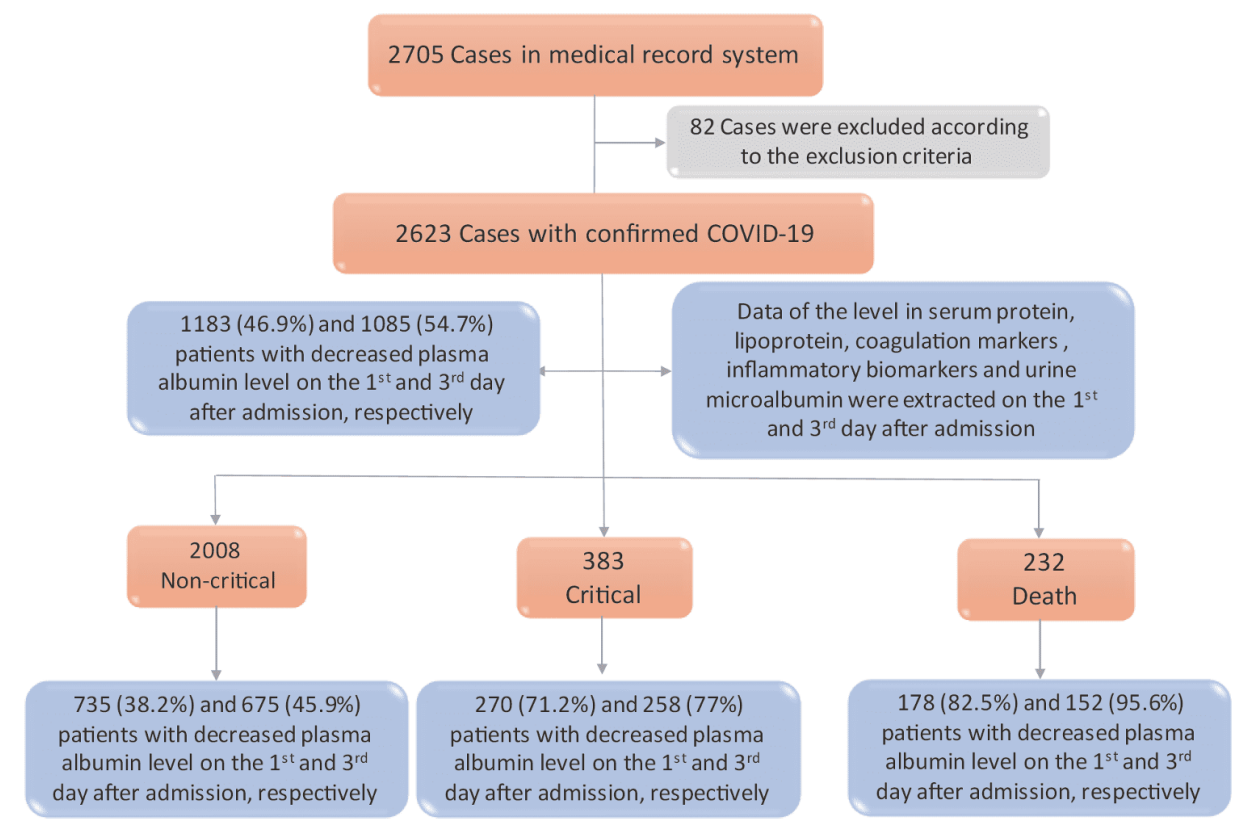

Figure 1 (Color online) Flowchart of patient recruitment.

Table 1 Baseline characteristics of patients ${ }^{a}$

\begin{tabular}{|c|c|c|c|c|}
\hline & Non-critically ill & Critically ill & Death & $P$ \\
\hline Age & $61.00(49.00,69.00)$ & $65.00(53.00,73.00)$ & $70.00(63.00,78.00)$ & $<0.001$ \\
\hline Gender (Female \%) & $1065(53.9)$ & $164(42.8)$ & $71(30.6)$ & $<0.001$ \\
\hline BMI & $23.53(21.48,25.48)$ & $24.09(22.04,25.83)$ & $24.37(22.63,26.57)$ & 0.177 \\
\hline Comorbidities & $710(42.9)$ & $208(65.0)$ & $137(69.5)$ & $<0.001$ \\
\hline Hyperlipidemia & $13(0.8)$ & $4(1.3)$ & $3(1.5)$ & 0.438 \\
\hline Body temperature $\left({ }^{\circ} \mathrm{C}\right)$ & $36.60(36.30,37.00)$ & $36.65(36.30,37.20)$ & $36.80(36.50,37.70)$ & $<0.001$ \\
\hline Heart rate, median (IQR) & $87.00(75.00,100.00)$ & $92.00(80.00,105.75)$ & $90.00(75.00,110.00)$ & $<0.001$ \\
\hline Respiratory rate, median (IQR) & $20.00(20.00,22.00)$ & $21.00(20.00,25.00)$ & $23.00(20.00,30.00)$ & $<0.001$ \\
\hline Mean SBP median (IQR), mmHg & $130.00(119.00,142.00)$ & $132.00(120.00,147.00)$ & $130.00(119.00,147.00)$ & 0.185 \\
\hline Mean DBP median (IQR), mmHg & $80.00(72.00,88.00)$ & $80.00(72.00,89.00)$ & $80.00(70.00,88.00)$ & 0.755 \\
\hline
\end{tabular}

a) Data are shown as median (IQR) or $n$ (\%). $P$ values were calculated by Kruskal-Wallis rank-sum test and Pearson's $\chi^{2}$ test as appropriate. BMI, body mass index.

duction in $\mathrm{A} / \mathrm{G}$ (ALB/GLO ratio) might be due to a decrease in albumin and slight increase in globulin (within the normal range). The total protein (TP) decreased in the three groups, which was probably caused by diarrhea and anorexia in COVID-19 patients (Figure 2D). In conclusion, albumin levels and $A / G$ ratio could be useful in the prognosis of COVID-19 patients. The lower the albumin level, the worse the prognosis (Table 2).

Meanwhile, the serum lipoproteins exhibited marked differences between patients in the non-critically ill, critically ill, and death groups. The level of low-density lipoprotein (LDL) in the death group (median, $1.92(1.43,2.52)$ ) was significantly lower than those in the critically ill (median, $2.09(1.60,2.68))$ and non-critically ill (median, 2.44 (1.96, 2.97)) groups (Figure $3 \mathrm{~A}$ ). The high-density lipoprotein (HDL) in the death group (median, 0.77 (IQR, 0.56, 0.92)) was also lower than that in the critically ill (median, 0.86 $(0.73,1.05))$ and non-critically ill (median, $2.44(0.80,1.16))$ groups (Figure 3B), which suggests impaired lipoprotein synthesis in COVID-19 patients. The triglyceride (TG) in the death group (median, 1.55 (IQR, 1.18, 2.21)) was also higher than that in the critically ill (median, $1.30(0.99,1.71)$ ) and non-critically ill (median, $1.25(0.96,1.77)$ ) groups (Figure 3C), suggesting an impaired TG and total cholesterol (TC) (Figure 3D) transportation caused by hepatocyte damage and reduction in HDL and LDL levels (Table 2).

\section{Analysis of urine protein loss in COVID-19 patients}

To identify the mechanism that causes albumin reduction, we examined the urine protein, mAlb, $\beta 2-\mathrm{MG}$, and $\alpha 1-\mathrm{MG}$. Results showed that only $11 \%$ of patients in the death group 


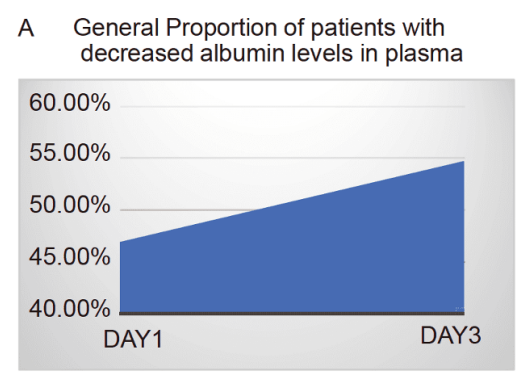

Days after admission

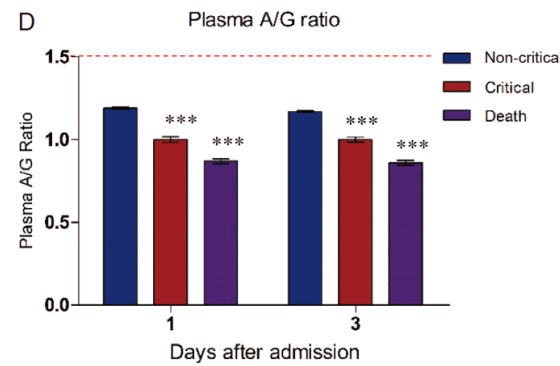

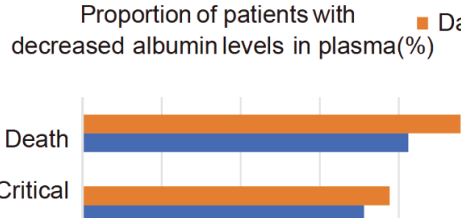

Non-critical

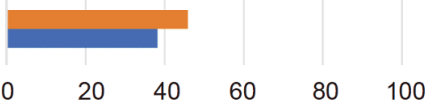

100

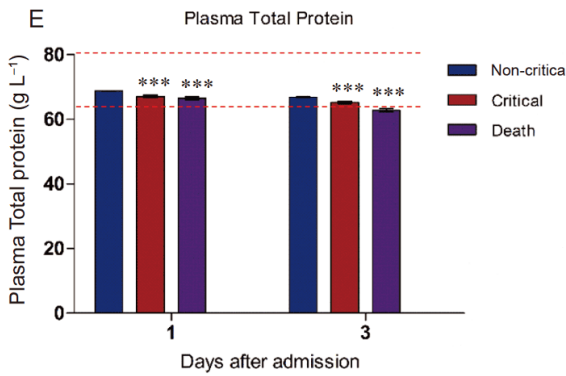

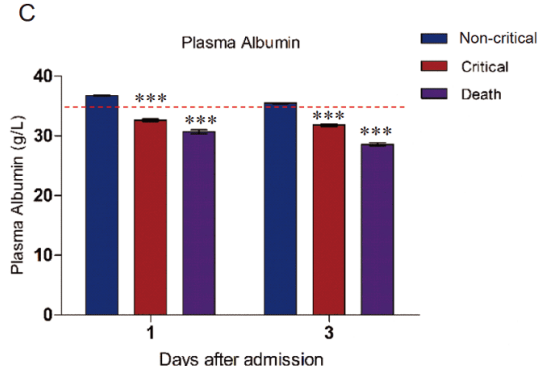

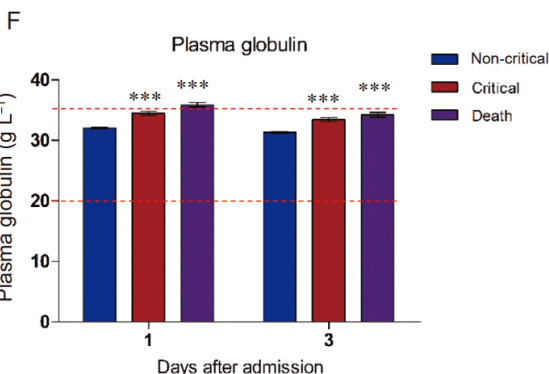

Figure 2 COVID-19 patients showed a decline in albumin and TP levels as the disease progressed. A, General proportion of patients with decreased albumin levels in serum during admission and after admission. B, The proportion of patients with decreased albumin level upon admission and after admission in the non-critical, critical, and death groups. C, The means of the albumin in serum during admission and after admission in the non-critical, critical, and death groups. D-F, The ratio of A/G and the level of total protein and serum globin in the three groups upon admission and after admission, respectively. The columns and error bars represent means and SEM. $* * * P<0.001$ were compared among non-critically ill, critically ill, and death groups. $P$ values were calculated by Kruskal-Wallis rank-sum test. The red dotted line indicates normal range.

Table 2 COVID-19 patients showed a decline in albumin and lipid synthesis as the severity of the disease developed ${ }^{\text {a) }}$

\begin{tabular}{|c|c|c|c|c|c|}
\hline & & Non-critically ill & Critically ill & Death & $P$ \\
\hline \multirow{2}{*}{ ALB (median (IQR)) } & At admission test & $36.60(33.18,40.40)$ & $32.20(29.60,35.75)$ & $31.10(27.85,34.20)$ & $<0.001$ \\
\hline & At second test & $35.50(32.40,38.50)$ & $31.30(28.80,34.52)$ & $28.80(25.40,31.35)$ & $<0.001$ \\
\hline \multirow{2}{*}{ GLO (median (IQR)) } & At admission test & $31.70(28.60,35.40)$ & $34.40(30.55,38.55)$ & $36.05(32.77,39.20)$ & $<0.001$ \\
\hline & At second test & $31.00(27.80,34.30)$ & $33.00(29.35,37.70)$ & $33.70(30.70,38.45)$ & $<0.001$ \\
\hline \multirow{2}{*}{ TP (median (IQR)) } & At admission test & $68.70(65.20,72.20)$ & $66.70(63.10,70.80)$ & $67.05(62.68,71.03)$ & $<0.001$ \\
\hline & At second test & $66.75(63.20,70.23)$ & $65.10(60.27,69.53)$ & $62.40(58.30,67.50)$ & $<0.001$ \\
\hline \multirow{2}{*}{ ALB/GLO (median (IQR)) } & At admission test & $1.16(0.96,1.38)$ & $0.93(0.79,1.10)$ & $0.84(0.74,0.98)$ & 0.558 \\
\hline & At second test & $1.15(0.97,1.34)$ & $0.94(0.79,1.10)$ & $0.84(0.71,0.98)$ & $<0.001$ \\
\hline \multirow{2}{*}{ TG (median (IQR)) } & At admission test & $1.25(0.96,1.77)$ & $1.30(0.99,1.71)$ & $1.55(1.18,2.21)$ & $<0.001$ \\
\hline & At second test & $1.48(1.09,2.10)$ & $1.48(1.07,2.02)$ & $1.69(1.25,2.31)$ & $<0.001$ \\
\hline \multirow{2}{*}{ HDL-C (median (IQR)) } & At admission test & $0.95(0.80,1.16)$ & $0.86(0.73,1.05)$ & $0.77(0.56,0.92)$ & $<0.001$ \\
\hline & At second test & $0.99(0.83,1.20)$ & $0.92(0.74,1.12)$ & $0.72(0.56,0.87)$ & $<0.001$ \\
\hline \multirow{2}{*}{ LDL-C (median (IQR)) } & At admission test & $2.44(1.96,2.97)$ & $2.09(1.60,2.68)$ & $1.92(1.43,2.52)$ & $<0.001$ \\
\hline & At second test & $2.50(2.02,2.95)$ & $2.29(1.68,2.83)$ & $1.84(1.36,2.47)$ & $<0.001$ \\
\hline
\end{tabular}

a) Data are shown as median (IQR). $P$ values were calculated by Kruskal-Wallis rank-sum test. The plasma levels of albumin (ALB), globulin (GLO), total protein (TP), and ALB/GLO were included as serum protein and triglyceride (TG), cholesterol (TC), HDL-C, and LDL-C levels upon admission and after admission. $P$ values were compared among non-critically ill, critically ill, and death groups. If the values of biomarkers were below the lower reference limit, half of the lower reference value was used.

(median, 210.70 (IQR, 84.65, 362.40) $\mathrm{mg} \mathrm{L}^{-1}$ ) and $12 \%$ of patients in the critically ill group (median, 19.10 (IQR, 6.12, 67.52) $\mathrm{mg} \mathrm{L}^{-1}$ ) had u-mAlb, which indicated only a small amount (19.1-362.4 mg/24 h) of albumin lost in the urine (Table 3). In summary, a small amount of urine protein loss in a very limited number of patients cannot justify the ex- tremely decreased albumin levels in the serum of a relatively large number of patients. Therefore, there may be disruption in the albumin synthesis.

We further analyzed the liver-related coagulation biomarkers, which demonstrated no significant extension in the prothrombin time (PT) (12-16) nor in the thromboplastin 

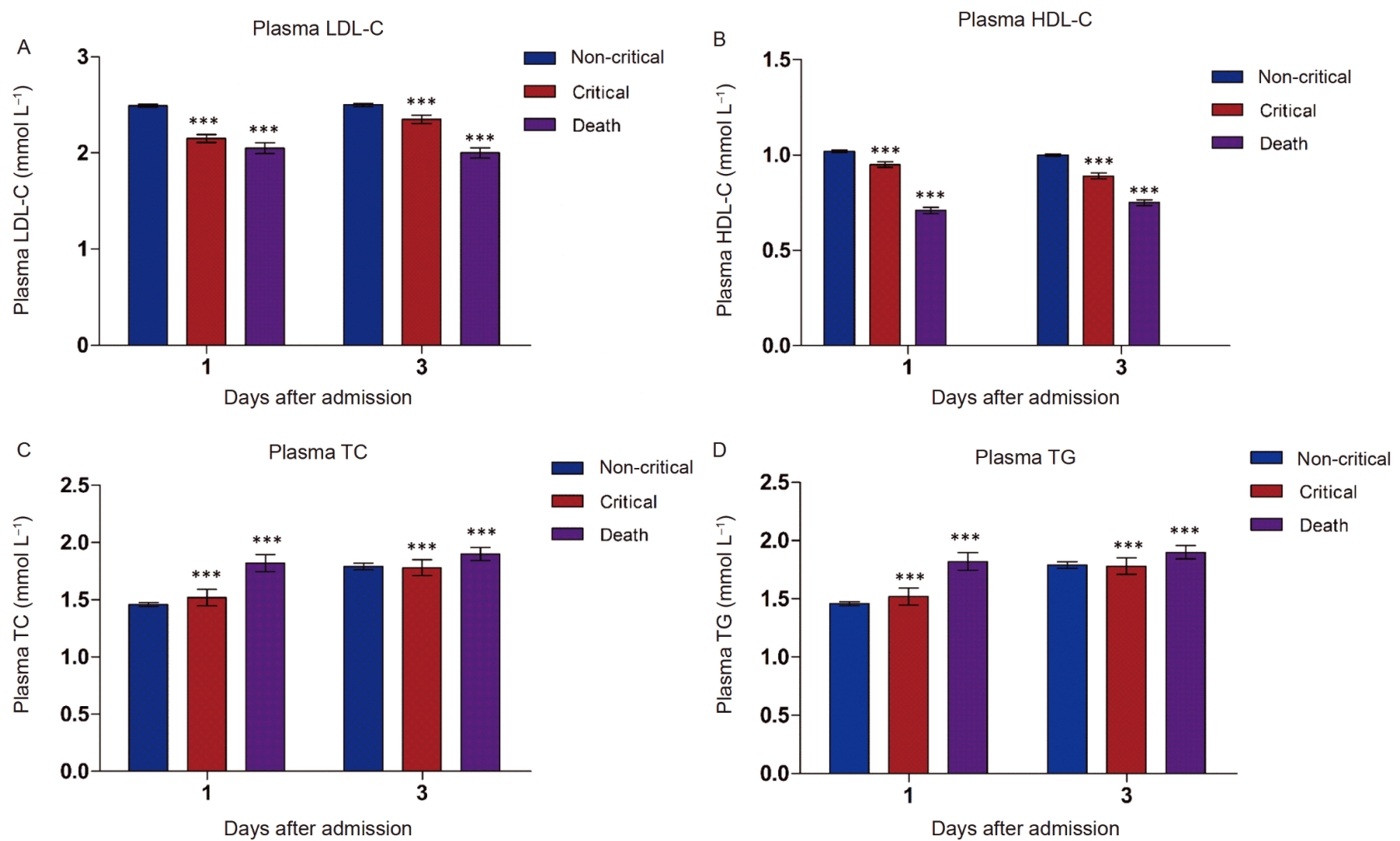

Figure 3 COVID-19 patients showed a decline in lipid synthesis as the severity of the disease developed. Panels A-D represent the lipoprotein of serum HDL-C, LDL-C, TC, and TG levels in the non-critically ill, critically ill, and death groups, respectively. The columns and error bars represent means and SEM. $* * * P<0.001$ were compared among non-critically ill, critically ill, and death groups. $P$ values were calculated by Kruskal-Wallis rank-sum test. The red dotted line indicates the normal range.

Table 3 Analysis of factors related to protein loss in COVID-19 patients ${ }^{\mathrm{a})}$

\begin{tabular}{cccccc}
\hline & & Non-critically ill & Critically ill & Death & $P$ \\
\hline \multirow{2}{*}{ MAlb (urine) (median (IQR)) } & At admission test & $5.55(3.00,11.92)$ & $19.10(6.12,67.52)$ & $210.70(84.65,362.40)$ & $<0.001$ \\
& At second test & $3.70(3.00,9.55)$ & $28.80(4.73,86.97)$ & $30.90(30.90,30.90)$ & $<0.001$ \\
\hline \multirow{2}{*}{$\beta 2-$ MG (median (IQR)) } & At admission test & $0.00(0.00,0.24)$ & $0.51(0.00,4.00)$ & $1.20(0.00,5.40)$ & $<0.001$ \\
& At second test & NA (NA, NA) & $2.75(1.85,5.38)$ & $0.00(0.00,0.00)$ & $<0.001$ \\
\hline \multirow{2}{*}{$\alpha 1$-MG-1 (median (IQR)) } & At admission test & $0.00(0.00,0.98)$ & $3.30(0.45,8.80)$ & $3.40(2.30,6.75)$ & $<0.001$ \\
& At second test & NA (NA, NA) & NA (NA, NA) & $0.00(0.00,0.00)$ & NA \\
\hline
\end{tabular}

a) Data are shown as median (IQR). $P$ values were calculated by Kruskal-Wallis rank-sum test. The urine microalbumin (u-mAlb), urine $\beta 2$-macroglobulin ( $\beta 2-\mathrm{MG})$, and $\alpha 1$-microglobulin ( $\alpha 1-\mathrm{MG})$ during admission and after admission were compared among non-critically ill, critically ill, and death groups. If the values of biomarkers were below the lower reference limit, half of the lower reference value was used.

time (TT) in the three groups, while the activated partial thromboplastin time (APTT) was prolonged in the critically ill and death groups, suggestive of reduced coagulation factors II, V, VIII, IX, XI, and XII. This observation also suggests that as an acute phase protein during the coagulation process, fiber Bragg grating (Fbg) stress increased in the acute inflammatory phase of liver invasion by the virus, and Fbg in all three groups was reduced to normal in the second test. Prothrombin activity (PTA) decreased with the exacerbation of the disease upon reexamination, which suggested that liver damage gradually increased (Table 4). This was also consistent with our hypothesis that the COVID-19 virus damages the ability of liver cells to synthesize albumin and some coagulation factors.

\section{Hepatic injury was closely related to the severity of COVID-19 patients}

The hepatic injury was generally mild. During the second observation, although the albumin level and $\mathrm{A} / \mathrm{G}$ had greatly reduced, the mean value of total bilirubin (TBil) serum reflecting the microstructural damage in liver cells had always been in the normal range. The sera aspartate aminotransferase (AST), alanine aminotransferase (ALT), and ALT/AST 
Table 4 COVID-19 patients have significant coagulopathy associated with significant liver dysfunction ${ }^{\text {a) }}$

\begin{tabular}{|c|c|c|c|c|c|}
\hline & & Non-critically ill & Critically ill & Death & $P$ \\
\hline \multirow{2}{*}{ TT (median (IQR)) } & At admission test & $16.50(15.70,17.40)$ & $16.70(15.90,17.80)$ & $17.10(15.90,19.10)$ & $<0.001$ \\
\hline & At second test & $16.80(16.00,17.70)$ & $16.60(15.50,18.10)$ & $17.50(16.30,20.20)$ & $<0.001$ \\
\hline \multirow{2}{*}{ PT (median (IQR)) } & At admission test & $13.70(13.20,14.20)$ & $14.30(13.50,15.20)$ & $15.30(14.30,16.90)$ & $<0.001$ \\
\hline & At second test & $13.50(13.10,14.00)$ & $14.10(13.40,14.90)$ & $16.25(14.83,18.10)$ & $<0.001$ \\
\hline \multirow{2}{*}{ APTT (median (IQR)) } & At admission test & $38.60(35.70,42.10)$ & $40.20(36.60,44.88)$ & $40.60(36.12,46.00)$ & $<0.001$ \\
\hline & At second test & $37.80(34.80,41.00)$ & $37.60(34.00,42.50)$ & $37.90(35.10,44.50)$ & $<0.001$ \\
\hline \multirow{2}{*}{ AT:A (median (IQR)) } & At admission test & $94.00(86.00,102.00)$ & $88.00(78.00,100.00)$ & $83.00(71.00,94.00)$ & $<0.001$ \\
\hline & At second test & $95.00(86.00,103.00)$ & $89.00(78.00,103.50)$ & $79.00(70.00,91.00)$ & $<0.001$ \\
\hline \multirow{2}{*}{ Fbg (median (IQR)) } & At admission test & $4.51(3.56,5.64)$ & $5.21(4.09,6.20)$ & $5.08(3.65,6.36)$ & $<0.001$ \\
\hline & At second test & $4.08(3.39,5.08)$ & $4.24(3.23,5.44)$ & $3.93(2.60,5.38)$ & $<0.001$ \\
\hline \multirow{2}{*}{ PTA (median (IQR)) } & At admission test & $93.00(86.00,100.00)$ & $86.00(76.00,95.00)$ & $75.00(64.00,86.00)$ & 0.167 \\
\hline & At second test & $94.00(87.00,102.00)$ & $88.00(78.00,97.00)$ & $67.00(55.00,79.00)$ & $<0.001$ \\
\hline
\end{tabular}

a) Data are shown as median (IQR). $P$ values were calculated by Kruskal-Wallis rank-sum test. The thromboplastin time (TT), prothrombin time (PT), activated partial thromboplastin time (APTT), AT:A, fibrinogen (Fbg), and prothrombin activity (PTA) upon admission and after admission were compared among non-critically ill, critically ill, and death groups. If the values of biomarkers were below the lower reference limit, half of the lower reference value was used.

were significantly increased in the critically ill and death groups. However, only the AST was mildly abnormal (41.00 $\left.(28.25,60.50) \mathrm{IU} \mathrm{L}^{-1}\right)$ in the death group during admission. It was worth noticing that the gamma-glutamyl transferase (GGT), a diagnostic biomarker for cholangiocyte injury, was significantly increased in the critically ill (median, 37.00 $(22.00,70.00))$ and death (median $39.00(25.00,70.00)$ ) groups compared to the non-critically ill group (median, $27.00(18.00,46.00))$ upon admission and increased more significantly in the second test in the non-critically ill group (29.00 (19.00, 49.00), critically ill group (38.00 (22.00, $78.00)$ ), and in the death group $(43.00(26.00,75.50))$ (Table $5)$. However, the hepatocyte injury was relatively mild. Patients often had no typical clinical symptoms of hepatitis. In a large cohort of 1,099 patients from 552 hospitals in 31 different regions in China, abnormal liver aminotransferase levels were more frequently observed in severe COVID-19 patients than in non-critical patients (Huang et al., 2020). Therefore, we speculated that the proportion of patients with transaminase abnormalities was high, but the transaminase abnormalities were mild, which indicated that liver cell necrosis seldom occurred. However, a decline in protein synthesis capacity, especially albumin synthesis, was induced by COVID-19 infection.

\section{The hepatic injury might be caused by COVID-19- induced cytokine storm}

The cytokines, including IL-6, IL-8, IL-10, IL-2R, IL-1 $\beta$, and TNF- $\alpha$, were significantly elevated at all levels in the three groups, which supports the notion that SARS-CoV-2 viruses trigger cytokine storm (Table S1 in Supporting Information). We analyzed the correlation of the changes in cytokines with liver damage. The $R$ values of GGT/IL-6, AST/IL-6, and ALT/IL-6 were $0.25,0.43$, and 0.16 $(P<0.001)$ (Figure $4 \mathrm{~A}-\mathrm{C})$, respectively, highlighting positive correlations between the levels of IL-6 and hepatic injury indicators (GGT, AST, and ALT). We further performed statistical analysis on the levels of cytokine (IL-6, IL-2R, and IL-8) and albumin. We extracted the data during admission and on days $1-3$, days $4-7$, days $8-14$, and $>14$ days after admission and analyzed the dynamic changes. We found that, upon the increase of IL-6, the albumin gradually decreased, indicating the progression of the disease. Interestingly, the IL-6 peak level basically coincided with the lowest level of albumin on the third day of admission, demonstrating the relationship between liver function damage caused by cytokine storms and albumin reduction (Figure 4D).

\section{DISCUSSION}

In this retrospective study, we discovered that a substantial number of COVID-19 patients exhibited significant reductions in the serum levels of albumin, LDL-C, and HDL-C. We also observed that patients with COVID-19 have decreased coagulation activity reflected by prolonged APTT. These findings suggest that protein synthesis in liver parenchymal cells was markedly inhibited. Importantly, reduced protein synthesis is strongly associated with the severity of COVID-19 and poor prognoses of patients. Furthermore, COVID-19 patients exhibited a surge in inflammatory factors, such as IL-6, IL-8, and IL-2 receptor (sST2) in serum, which are related to the inhibition of liver functions and severity of the disease.

The liver has a strong compensatory ability. Mild liver 
Table 5 The hepatic injury is closely related to the severity of COVID-19 patients ${ }^{\text {a) }}$

\begin{tabular}{cccccc}
\hline & & Non-critically ill & Critically ill & Death & $P$ \\
\hline \multirow{2}{*}{ ALT (median (IQR)) } & At admission test & $21.00(14.00,37.00)$ & $25.00(15.00,40.00)$ & $28.00(18.00,43.00)$ & $<0.001$ \\
& At second test & $24.00(16.00,42.00)$ & $28.00(17.00,46.00)$ & $27.00(17.00,44.00)$ & $<0.001$ \\
\hline \multirow{2}{*}{ AST (median (IQR)) } & At admission test & $24.00(18.00,34.00)$ & $33.00(23.00,49.00)$ & $41.00(28.25,60.50)$ & $<0.001$ \\
& At second test & $21.00(16.00,29.00)$ & $25.00(18.00,37.00)$ & $35.00(24.00,53.50)$ & $<0.001$ \\
\hline \multirow{2}{*}{ GGT (median (IQR)) } & At admission test & $27.00(18.00,46.00)$ & $37.00(22.00,70.00)$ & $39.00(25.00,70.00)$ & $<0.001$ \\
& At second test & $29.00(19.00,49.00)$ & $38.00(22.00,78.00)$ & $43.00(26.00,75.50)$ & $<0.001$ \\
\hline \multirow{2}{*}{ ALT/AST (median (IQR)) } & At admission test & $0.71(0.56,0.93)$ & $0.67(0.60,1.08)$ & $0.60(0.53,0.77)$ & 0.558 \\
& At second test & $0.97(0.66,1.38)$ & $1.05(0.70,1.33)$ & $0.56(0.56,0.56)$ & 0.455 \\
\hline \multirow{2}{*}{ TBIL (median (IQR)) } & At admission test & $8.30(6.20,11.40)$ & $10.00(7.30,14.50)$ & $12.20(8.60,17.60)$ & $<0.001$ \\
& At second test & $8.20(6.30,11.30)$ & $10.50(7.62,14.57)$ & $13.70(9.35,20.30)$ & $<0.001$ \\
\hline \multirow{2}{*}{ DBIL (median (IQR)) } & At admission test & $3.60(2.60,4.80)$ & $4.70(3.45,6.70)$ & $5.85(4.20,9.22)$ & $<0.001$ \\
& At second test & $3.40(2.60,4.60)$ & $4.70(3.50,7.00)$ & $6.90(4.50,11.00)$ & $<0.001$ \\
\hline \multirow{2}{*}{ IBIL (median (IQR)) } & At admission test & $4.70(3.40,6.70)$ & $5.10(3.58,7.73)$ & $5.75(4.10,8.33)$ & $<0.001$ \\
& At second test & $4.80(3.60,6.70)$ & $5.50(3.90,7.80)$ & $6.20(3.88,9.62)$ & $<0.001$ \\
\hline
\end{tabular}

a) Data are shown as median (IQR). $P$ values were calculated by Kruskal-Wallis rank-sum test. The levels of alanine aminotransferase (ALT), aspartate aminotransferase (AST), $\gamma$-glutamyl transpeptidase (GGT), AST/ALT, total bilirubin (TBil), direct bilirubin (DBil), and indirect Bilirubin (IBiL) on admission and after admission were compared among non-critically ill, critically ill, and death groups. If the values of biomarkers were below the lower reference limit, half of the lower reference value was used.

damage will not cause selective decrease in the serum albumin level (Mao et al., 2020). In this study, we found that the proportion of hypoalbuminemia $\left(<35 \mathrm{~g} \mathrm{~L}^{-1}\right)$ accounted for $49.6 \%$ of all patients on admission and further decreased to $39.4 \%$ after hospitalization. For patients with persistent hypoalbuminemia, the serum albumin level became even lower after hospitalization than that during admission, suggesting that liver damage in this group of patients was exacerbated as the disease progressed.

Furthermore, the decrease in serum albumin levels might result from mild diarrhea following the onset of the disease (Murthy et al., 2020). In our cohort, however, we found no significant difference in the frequency of diarrhea among three groups during the observational period; therefore, the decrease in albumin levels cannot be elucidated by the loss of albumin from the intestine. The intake of nutrients is unlikely the cause, either, because patients already had reduced albumin levels upon admission to the hospital. For patients under critical conditions, their serum albumin levels could not be normalized by daily intravenous supply of serum or albumin. Renal damage can be largely excluded, given that the patients have very small amount of albumin in the urine. Therefore, we extrapolate that hepatocyte dysfunction in albumin synthesis is likely to be the major cause of hypoalbuminemia. This conclusion is supported by facts that the patients display, such as (i) reduced LDL-C and HDL-C level and (ii) coagulation dysfunction. Although hypoalbuminemia could also be attributed to vascular leakage of tissues and organs, such as the overwhelming inflammatory responses in pathological manifestation of COVID-19 in the lungs of COVID-19 patients, it appears not to support hy- poalbuminemia that resulted from extensive tissue edema, as massive macrophage infiltration and mucinous secretion in the bronchioles likely occur prior to serous and fibrinoid exudation in the alveoli. Moreover, the pulmonary edema is a pathological change of every end stage (Wang, 2020).

Critical hypoalbuminemia is associated with poor prognosis and deserves clinical intervention. Thus, it is important to understand how SARS-CoV-2 viruses injure protein synthesis of hepatocytes. One explanation is that SARS-CoV-2 might infect the liver and induce hepatitis. However, this hypothesis is not supported by histologic results, which did not suggest hepatitis (Xu et al., 2020), with an almost normal serum levels of glutamic oxaloacetic transaminase (GOT) and glutamicpyruvic transaminase. Notably, cytokine storm develops in critical COVID-19 patients (Sun et al., 2020). In our cohort, a broad spectrum of inflammatory factors was tested, and a bundle of cytokines, including IL-1, IL-6, IL-8, and IL2R, as well as classical inflammatory biomarker C-reactive protein, dramatically increased. It is important to note that the levels of inflammatory cytokines are inversely correlated with albumin level. Therefore, the most likely mechanism is that the upsurge of inflammatory cytokines results in the inhibition of protein synthesis in hepatocytes (Imaeda et al., 2009) rather than viral hepatitis. Although the viral receptor, angiotensin-converting enzyme 2 (ACE2), can be detected in certain types of cells in the liver (Chai et al., 2020), the liver damage directly from the viruses would alter the enzymes first and then affect albumin, lipoproteins, and coagulation functions, only if the viral infection prevailed in the liver. Moreover, the reduction of albumin correlates well with the surge of cytokines, further supporting our notion that liver injury is immediately sec- 

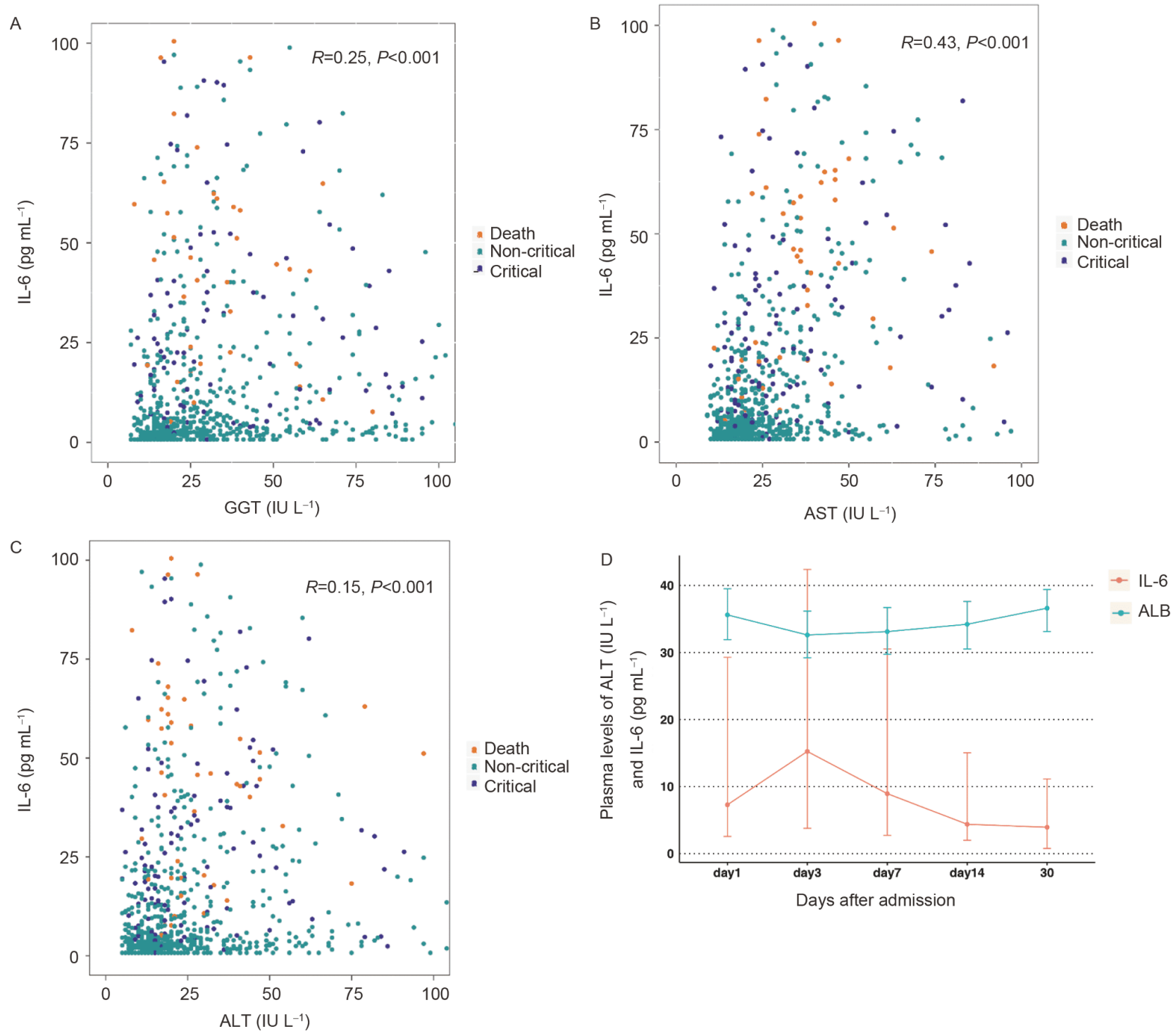

Figure 4 Correlations of GGT, ALT, and AST with IL-6 during admission. A, GGT and IL-6. B, AST and IL-6. C, ALT and IL-6. D, The fluctuation of the serum levels of Alb and IL- 6 on the 1st, 3rd, 7th, and 14th day and $>14$ days after admission. ALT, alanine aminotransferase. AST, aspartate aminotransferase. GGT, $\gamma$-glutamyl transpeptidase. IL-6, interleukin-6. Alb, albumin. Spearman correlations were calculated between IL-6 level and GGT, AST, and ALT levels, respectively. A two-sided $P$ value below 0.05 was considered statistically significant.

ondary to cytokine storm. But which cytokine(s) and how they injure liver functions need detailed studies in the future to be ruled out.

In summary, our study demonstrated that some patients with COVID-19 have distinct hypoalbuminemia, which is likely due to hepatotoxicity of cytokine storm. Hypoalbuminemia is associated with poor prognosis of patients, and intravenous supplements of serum or albumin should be a therapeutic strategy to improve clinical outcomes (Figure 5).

\section{METHODS}

\section{Study design and oversight}

This study was a retrospective analysis involving 2,623
COVID-19 patients between January 29, 2020 and March 6, 2020 in Tongji Hospital, Wuhan, China, and was approved by the institutional review board of Tongji Hospital ethics committee (TJ-IRB20200229). The requirement for informed consent was waived because the data are anonymous.

\section{Data collection}

The healthcare data regarding clinical manifestations, coexisting diseases, laboratory results, and survival status were collected by data coordinators through the electronic medical chart. Coexisting diseases were defined as preexisting diagnosis prior to hospital admission reported by patients themselves. The laboratory results, including inflammation biomarkers and hepatic injury biomarkers, albumin, lipo- 


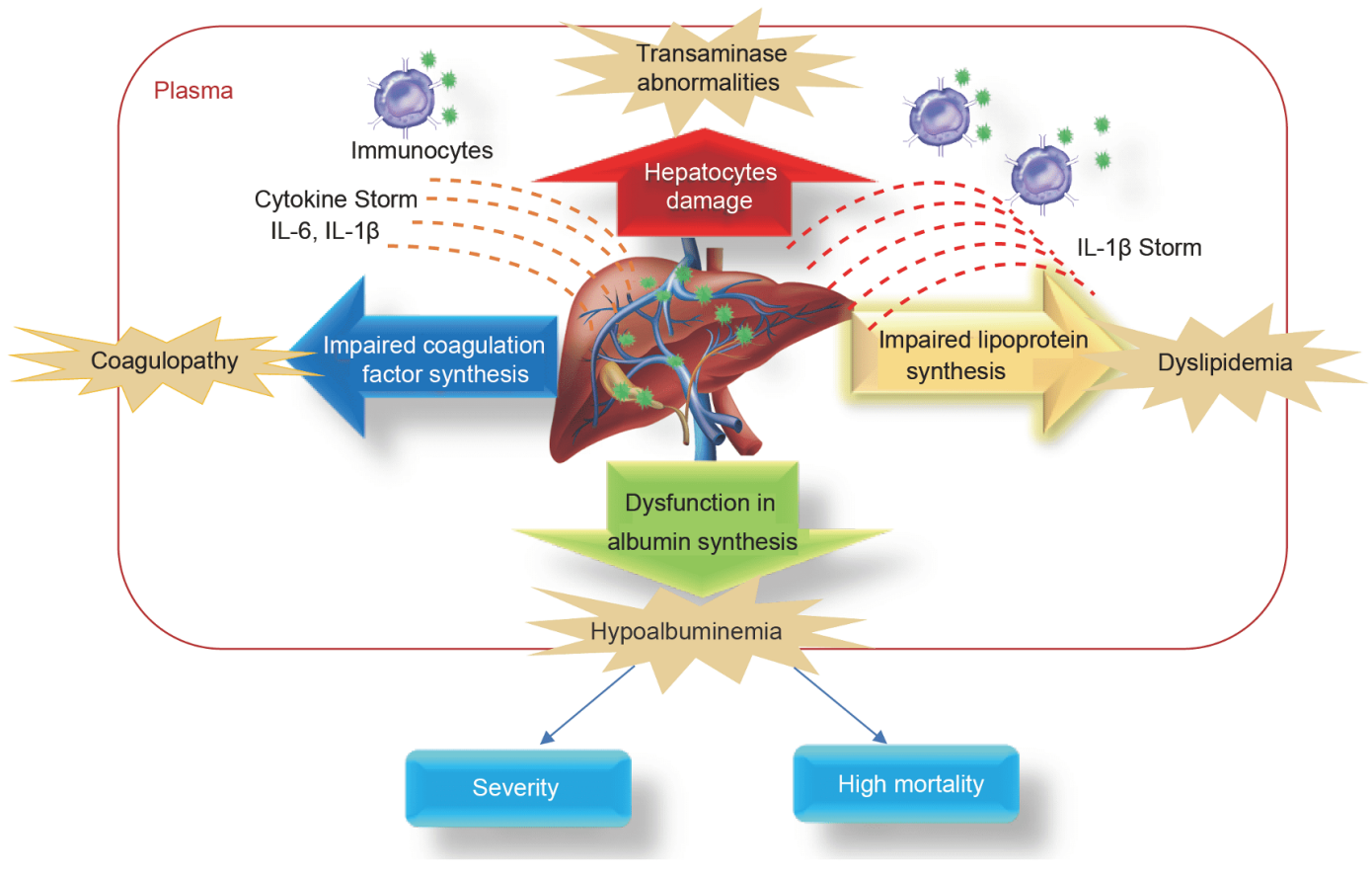

Figure 5 (Color online) The working model of albumin decrease in COVID-19 patients. Patients with COVID-19 have marked hypoalbuminemia due to hepatoxic effects of cytokine storm, which is associated with poor prognosis.

proteins, urine microalbumin ( $\mathrm{u}-\mathrm{Alb}$ ), and urine $\beta 2$-macroglobulin $(\beta 2-\mathrm{MG})$ and $\alpha 1$-microglobulin $(\alpha 1-\mathrm{MG})$ were extracted during admission and at the second test.

\section{Study population}

Patients included in this study were those diagnosed with COVID-19, according to the Clinical Guidance for COVID19 and Diagnosis and Treatment published by the National Health Commission of China. All patients have typical medical history and imaging characteristics of COVID-19. Patients younger than 18 years old, had acute hepatitis within one month, and with history of liver or renal dysfunction were excluded from this study. According to the Clinical Guidance for COVID-19 Diagnosis and Treatment published by the National Health Commission of China, all COVID-19 patients were divided into four types: mild, general, severe, and critical COVID-19 (National Health Commission of China, 2020). However, in this study, we only recruited general, severe, and critical COVID-19 patients. All recruited patients were further categorized into three groups: non-critically ill (general and severe), critically ill (survival), and death. Of these, patients belonging to the critically ill group met one of the following criteria: (i) patients had respiratory failure and needed mechanical ventilation; (ii) patients experienced shock during hospitalization; or (iii) patients with other organ failures required intensive care unit monitoring and treatment. Furthermore, according to clinical outcomes, patients were identified as critically ill survivors if they survive until March 6, 2020, or discharged before
March 6, 2020; otherwise, they will fall under death group.

\section{Biomarkers}

All serum biomarkers wildly used in the clinical practice were measured under standard hospital assays, including (i) activity of liver enzymes: alanine aminotransferase (ALT), aspartate aminotransferase (AST), $\gamma$-glutamyl transpeptidase (GGT), AST/ALT, and total bilirubin (TBil), direct bilirubin (DBil), and indirect Bilirubin (IBiL) as hepatic biomarker; (ii) serum protein level: albumin, globulin (GLO), TP, ALB/ GLO, as well as triglyceride (TG), TC, and HDL-C, LDL-C are included as lipoprotein; (iii) TT, prothrombin time (PT), activated partial thromboplastin time (APTT), antithrombin activity (AT:A), fibrinogen (Fbg), prothrombin activity (PTA), and D-dimer level were considered as biomarkers of coagulation; (iv) interleukin-6 (IL-6), interleukin-8 (IL-8), interleukin-10 (IL-10), interleukin-2 receptor (IL-2R), interleukin-1 beta (IL-1 $\beta$ ), and tumor necrosis factor $\alpha$ (TNF$\alpha$ ) were used as inflammatory biomarkers. Additionally, umAlb, $\beta 2-M G$, and $\alpha 1-\mathrm{MG}$ were included as markers of protein loss in urine. The level change of these biomarkers was identified based on chemical laboratory criteria. These measures were recorded on the day of hospital admission and, thereafter, retested on the following days.

\section{Statistical analysis}

The continuous values were shown as median and interquartile range and the categorical variables as counts and 
percentages. The Kruskal-Wallis rank-sum test was utilized to evaluate the differences in non-normal distributed continuous values, and the differences in categorical variables were evaluated under the Pearson's $\chi^{2}$ test. Spearman correlations were calculated between IL- 6 level and GGT, AST, and ALT levels, respectively. The OR and 95\% CI were calculated by multivariable regression models. A two-sided $P$ value below 0.05 was considered statistically significant. All statistical analyses were performed using $\mathrm{R}$ packages (version 3.1.4, Vienna, Austria).

\section{Definitions}

All biomarkers were measured using standard assays run by the Department of Laboratory Medicine of the hospital. Coagulopathy was defined as a three-second prolongation of PT or a five-second prolongation of APTT. Hypoproteinemia was defined as albumin $<35 \mathrm{~g} \mathrm{~L}^{-1}$ serum. According to the manufacturers' instructions, the threshold of transaminase was defined as AST and ALT $<40 \mathrm{IU} \mathrm{L}^{-1}$ and ALT/AST ratio between 0.8 and 1.5. The normal ranges of serum lipids were defined as TC $2.8-5.17 \mathrm{mmol} \mathrm{L}^{-1}$, TG $2.8-5.17 \mathrm{mmol} \mathrm{L}^{-1}$, LDL 2.8-5.17 $\mathrm{mmol} \mathrm{L}^{-1}$, and HDL $0.78-2.0 \mathrm{mmol} \mathrm{L}^{-1}$. The normal range of IL-6, IL-8, IL-10, IL-2R, IL-1 $\beta$, and TNF- $\alpha$ were below $7,62,9.1,710,5$, and $8.1 \mathrm{pg} \mathrm{mL}^{-1}$, respectively. Day 1 means the first test upon admission, and day 3 means repeat tests on the following days (the interval between these two testing ranges from 3 to 14 days based on the severity of the disease. Most of the retests were performed on the third to fourth day after admission).

Compliance and ethics The author(s) declare that they have no conflict of interest.

Acknowledgements This work was supported in part by the National Natural Science Foundation of China (81790624, 81630010, and 81800261).

\section{References}

Arentz, M., Yim, E., Klaff, L., Lokhandwala, S., Riedo, F.X., Chong, M., and Lee, M. (2020). Characteristics and outcomes of 21 critically ill patients with COVID-19 in Washington state. JAMA 323, 1612.

Chai, X., Hu, L., Zhang, Y., Han, W., Lu, Z., Ke, A., Zhou, J., Shi, G., Fang, N., Fan, J., et al. (2020). Specific ACE2 expression in cholangiocytes may cause liver damage after 2019-nCoV infection. bioRxiv, https://doi.org/10.1101/2020.02.03.931766.

Chen, C., Zhou, Y., and Wang, D.W. (2020). SARS-CoV-2: a potential novel etiology of fulminant myocarditis. Herz 45, 230-232.
Guan, W., Ni, Z., Hu, Y., Liang, W., Ou, C., He, J., Liu, L., Shan, H., Lei, C., Hui, D.S.C., et al. (2020). Clinical characteristics of coronavirus disease 2019 in China. N Engl J Med 382, 1708-1720.

Holshue, M.L., DeBolt, C., Lindquist, S., Lofy, K.H., Wiesman, J., Bruce, H., Spitters, C., Ericson, K., Wilkerson, S., Tural, A., et al. (2020). First case of 2019 novel coronavirus in the United States. N Engl J Med 382, 929-936.

Huang, C., Wang, Y., Li, X., Ren, L., Zhao, J., Hu, Y., Zhang, L., Fan, G., $\mathrm{Xu}, \mathrm{J} ., \mathrm{Gu}, \mathrm{X}$., et al. (2020). Clinical features of patients infected with 2019 novel coronavirus in Wuhan, China. Lancet 395, 497-506.

Imaeda, A.B., Watanabe, A., Sohail, M.A., Mahmood, S., Mohamadnejad, M., Sutterwala, F.S., Flavell, R.A., and Mehal, W.Z. (2009). Acetaminophen-induced hepatotoxicity in mice is dependent on Tlr9 and the Nalp3 inflammasome. J Clin Invest 119, 305-314.

Liu, Y., Yan, L.M., Wan, L., Xiang, T.X., Le, A., Liu, J.M., Peiris, M., Poon, L.L.M., and Zhang, W. (2020a). Viral dynamics in mild and severe cases of COVID-19. Lancet Infect Dis https://doi.org/10.1016/ S1473-3099(20)30232-2.

Liu, Y., Yang, Y., Zhang, C., Huang, F., Wang, F., Yuan, J., Wang, Z., Li, J., Li, J., Feng, C., et al. (2020b). Clinical and biochemical indexes from 2019-nCoV infected patients linked to viral loads and lung injury. Sci China Life Sci 63, 364-374.

Mao, R., Liang, J., Shen, J., Ghosh, S., Zhu, L.R., Yang, H., Wu, K.C., and Chen, M.H. (2020). Implications of COVID-19 for patients with preexisting digestive diseases. Lancet Gastroenterol Hepatol 5, 425-427.

Matthay, M.A., Aldrich, J.M., and Gotts, J.E. (2020). Treatment for severe acute respiratory distress syndrome from COVID-19. Lancet Respir Med 8, 433-434.

Murthy, S., Gomersall, C.D., and Fowler, R.A. (2020). Care for critically ill patients with COVID-19. JAMA 323, 1499.

National Health Commission of China. (2020). Chinese management guideline for COVID-19 (version 5.0). http://wwwnhcgoven/yzygj/ s7653p/202002/d4b895337e19445f8d728fcaf1e3e13a.shtml.

Peiris, J.S.M., Guan, Y., and Yuen, K.Y. (2004). Severe acute respiratory syndrome. Nat Med 10, S88-S97.

Saghazadeh, A., and Rezaei, N. (2020). Immune-epidemiological parameters of the novel coronavirus - a perspective. Expert Rev Clin Immunol https://doi.org/10.1080/1744666X.2020.1750954.

Shi, S., Qin, M., Shen, B., Cai, Y., Liu, T., Yang, F., Gong, W., Liu, X., Liang, J., Zhao, Q., et al. (2020). Association of cardiac injury with mortality in hospitalized patients with COVID-19 in Wuhan, China. JAMA Cardiol https://doi.org/10.1001/jamacardio.2020.0950.

Sun, D., Li, H., Lu, X.X., Xiao, H., Ren, J., Zhang, F.R., and Liu, Z.S. (2020). Clinical features of severe pediatric patients with coronavirus disease 2019 in Wuhan: a single center's observational study. World J Pediatr https://doi.org/10.1007/s12519-020-00354-4.

Wang, C., Xie, J., Zhao, L., Fei, XC., Zhang, H., Tan, Y., Zhou, LT., Liu, ZH., Ren, Y., Yuan, L., et al. (2020). Aveolar macrophage activation and cytokine storm in the pathogenesis of critical COVID-19. Research Square, https://doi.org/10.21203/rs.3.rs-19346/v1.

Wilder-Smith, A., Chiew, C.J., and Lee, V.J. (2020). Can we contain the COVID-19 outbreak with the same measures as for SARS? Lancet Infect Dis 20, e102-e107.

Xu, Z., Shi, L., Wang, Y., Zhang, J., Huang, L., Zhang, C., Liu, S., Zhao, P., Liu, H., Zhu, L., et al. (2020). Pathological findings of COVID-19 associated with acute respiratory distress syndrome. Lancet Respir Med $8,420-422$.

\section{SUPPORTING INFORMATION}

The supporting information is available online at https://doi.org/10.1007/s11427-020-1733-4. The supporting materials are published as submitted, without typesetting or editing. The responsibility for scientific accuracy and content remains entirely with the authors. 